\title{
Determining of optimal telemedicine communication technologies with regards to network interactive modes: A Delphi survey
}

\author{
Mostafa Shanbehzadeh1(i), Hadi Kazemi-Arpanahi2, 3*(D), Mohammad Mahbubi ${ }^{4}$ \\ ${ }^{1}$ Assistant Professor, Department of Health Information Technology, School of Paramedical, Ilam University of Medical Sciences, Ilam, Iran \\ ${ }^{2}$ Assistant Professor, Department of Health Information Technology, Abadan Faculty of Medical Sciences, Abadan, Iran \\ ${ }^{3}$ Student Research Committee, Abadan Faculty of Medical Sciences, Abadan, Iran \\ ${ }^{4}$ Associate Professor, Department of public Health, Abadan Faculty of Medical Sciences, Abadan, Iran
}

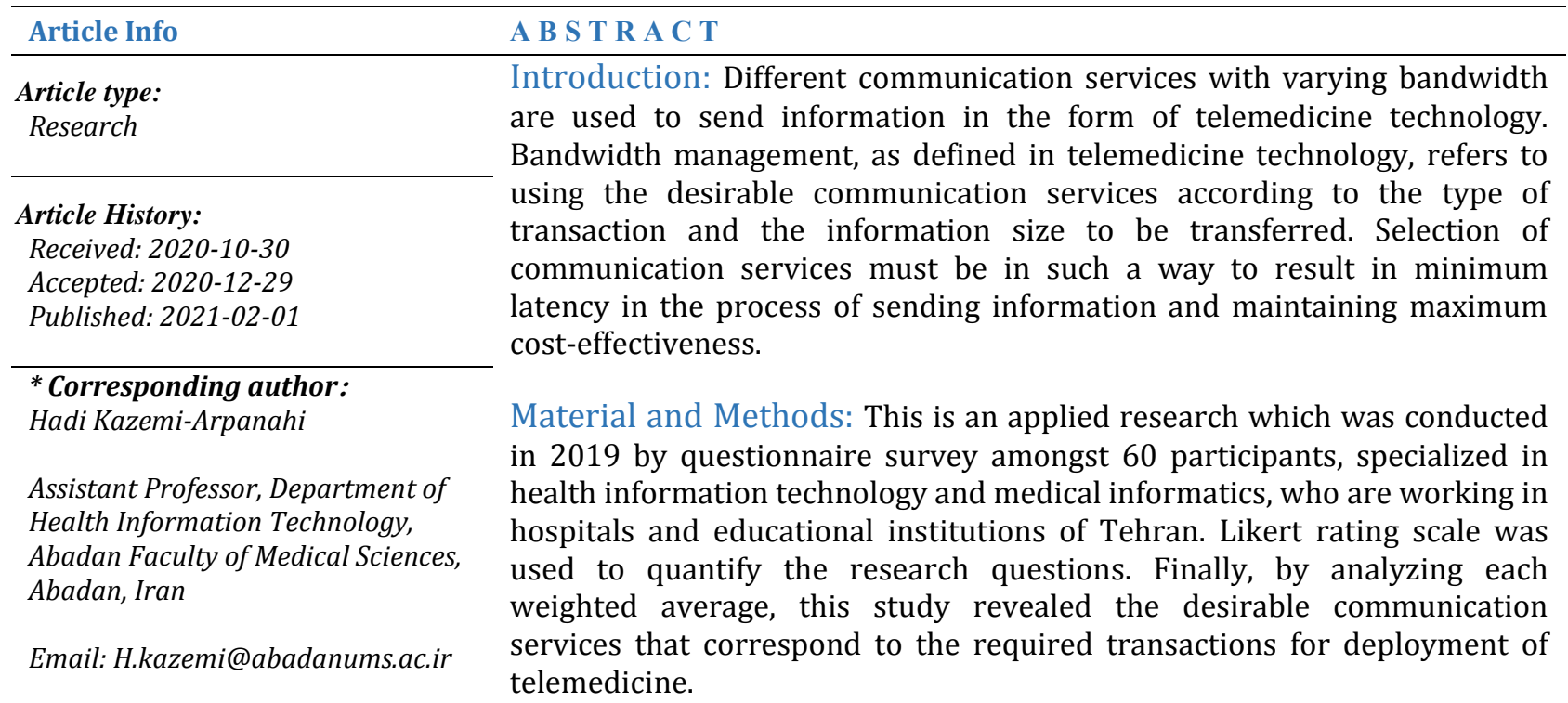

Keywords:

Telemedicine

Bandwidth

Interactive Modes

Communication Services
Results: Transfer of multimedia information, using synchronized teleconferencing via primary low bandwidth technologies, had the lowest number average (0.96) and transmission of hybrid data (combination of picture, text, multimedia templates in synchronized or asynchronized modes) via Asymmetric Digital Subscriber Line (ADSL) technology had the highest average (4.96).

Conclusion: Selection of communication services, with regard to its convergence with the information size and the type of their application, plays a significant role in controlling network traffic and preventing latency in the process of sending information in the context of telemedicine technology. High bandwidth communication services should be used for those telemedicine systems, which are offering services to many users, as well as those in which real-time transmission of information is essential. It needs to be pointed out that with regard to the cost-effectiveness of sending information, it is necessary to use low-cost services with low bandwidth for transfer of light weight information as well as for asynchronous applications in which latency in the process of information transfer is not detrimental.

Cite this paper as:

Shanbehzadeh M, Kazemi-Arpanahi H, Mahbubi, M. Determining of Optimal Telemedicine Communication Technologies with Regards to Network Interactive Modes: A Delphi Survey. Front Health Inform. 2021; 10: 58. DOI:

$\underline{10.30699 / f h i . v 10 \mathrm{i} 1.256}$

\section{INTRODUCTION}

Due to the rapid advances in information technology, homes and workplaces all over the world have undergone fundamental changes. These changes can also be noticed in the field of healthcare and health system $[\underline{1}, \underline{2}]$. Application of health information technology, especially in hospitals and health centers develops great potential to enhance the quality of provided services as well as efficiency and effectiveness of the personnel $[\underline{3}, \underline{4}]$. In various ways, health information technology can be effective 
in the field of medicine. Telemedicine networks, patient's electronic records and health information networks are some examples of the application of information technology in the field of health [ $\underline{5}-7]$. One of the most important events related to technology in late twentieth century was the cooccurrence of human development and telecommunication emergence. Telemedicine as a subtype of telecommunication deals with the use of technology in the field of medicine to improve healthcare [ $[-10]$. In the definition of telemedicine, the World Health Organization has adopted the following description: "The delivery of health care services, where distance is a critical factor, by all health care professionals using information and communication technologies for the exchange of valid information for diagnosis, treatment and prevention of disease and injuries, research and education, and for the continuing education of health care providers, all in the interests of advancing the health of individuals and their communities". In another definition, telemedicine is defined as the proper transfer of images and health data, for the purpose of providing the long-distance patients with better healthcare services [11- $\underline{13}]$.

Virtual care delivery can synchronous or asynchronous. Each mode has different IT infrastructure requirements. Synchronous telemedicine involves virtual meetings that happen in timely manner, usually involving two-way interaction using audio and video to avoid the need for an in-person visit. Synchronous meetings should be used when conversation is necessary, such as during new patient consultations, preoperative counseling, postoperative assessments, and followup visits. This session can be conducted for both new and established patients as well as consults. Asynchronous telemedicine involves the collecting, brief, storing, and exchanging of data for a patient or, more often, another provider to review at a later

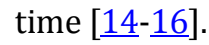

In order to transmit the information in telemedicine format, a set of technologies and communication services with varying bandwidth are used including Plain Old Telephone Services (POTS), Integrated Services Digital Network (ISDN), Digital Subscriber Line (DSL), Asynchronous Transfer Mode (ATM), Satellite and Wireless Technology [17]. In a report entitled as "Telecommunication Requirements in Telemedicine", communication technologies and information transmitters are categorized based on their bandwidths in five categories as follows:

- Plain old telephone services, via typical telephone lines and modem services, enables transfer of textual information, laboratory reports and findings from physiological measures in the format of store-and-forward technologies, such as email, fax according to the low bandwidth $(18,36$, and $54 \mathrm{~kb} / \mathrm{s})$ [18].

- Integrated services digital network was the first type of information digital transfer, which enables the rapid and timely transport of text, video and sometimes voice on-demand. Its bandwidth variably ranges from $128 \mathrm{Kbps}$ in ISDN Basic Rate to 1.5 Mbps in ISDN Primary Rate [17].

- Digital subscriber lines, the regular telephone lines are used to transmit information with reference to the high bandwidth. This technology has five subcategories, i.e. (1) Very High data rate Digital Subscriber Line (VDSL) (1.5 to 52 Mbps); (2) Symmetric Digital Subscriber Line (SDSL), (2.3 Mbps); (3) Asymmetric Digital Subscriber Line (ADSL) (640 Kbps to 8Mbps); (4) Integrated Services Digital Network (ISDN) of the Digital Subscriber Line (DSL) type (144 Kbps); (5) High bit rate Digital Subscriber (HDSL) (1.544 Mbps) $[19,20]$.

- Asynchronous transfer mode is more efficient, when considering the inefficiency of integrated digital network in real-time transfer of larger information packets in multimedia format, rating from 8 to 155 Mbps and even up to $9 \mathrm{Gbps}$. In one report, these communication services are introduced as one of the communication technologies in telemedicine, which have an ultrahigh bandwidth. This technology is reported to be suitable for transporting information in companies where different users are provided with telemedicine services as backbone. Moreover, this technology also appears to be suitable for sending video information in remote surgeries, requiring high resolution imaging $[\underline{21}, \underline{22}]$.

- Satellite broadcasting technology allows continuous and flexible access to telemedicine, even in secluded areas. This paves the way for swift response in crises and emergencies. A bandwidth $512 \mathrm{Kbps}$ to 1 Mbps is required since this technology uses radio waves with short wavelength to transfer the released information [ $\underline{23}-\underline{25}]$.

- Other communication technologies in telemedicine include wireless technology as well as internet technology. Wireless technologies are used to instantly send the patient's record in emergencies via third generation mobile services, WiFi, WiMax and radio-frequency identification technology with a relatively high bandwidth 
(300 Kbps). Internet , as the latest development in telemedicine communication technology, is more widely used in comparison to other technologies, that allows transfer of information via the World Wide Web over high bandwidth (10 Mbps) [26].

To send information in telemedicine, a number of interactive methods, with regard to the nature of related transaction, are used. These methods are categorized in four major groups as follows:

1. Transferring text files in electronic-mail format;

2. Transferring images and biological data in the format of store-and-forward technology;

3. Transferring Voice Over Internet Protocol (VOIP);

4. Transferring information in video conference format [27-31].

The exchanged information via telemedicine has a variety of problems; and subsequently the size of each can be different. In one categorization, the information is categorized in an ascending order of their size as follows:

1. Textual information (health reports);

2. Numerical-textual information (laboratory findings and other clinical measurements);

3. Images (radiology, pathology, and skin lesions images);

4. Audio (heartbeat sounds and telephone conversations);

5. Multimedia (the video teleconferencing with the purpose of consultation and distance teaching, videos of remote surgery) [30-33].

As it was pointed out, each of the type of information that are sent in telemedicine format has a particular size, which typically require different bandwidth technologies for transmission. This bandwidth should be chosen according to the type of the information, their size, and the number of users in telemedicine systems. In telemedicine systems, maintaining the cost-effectiveness of information transfer is essential. Hence, to this effect, it would be better to select the communication technologies, following the interactive manner. Bandwidth management is a term used to refer to the process of adopting a flexible approach by means of classifying the information according to their size and subsequently selecting the appropriate bandwidth, proportional to the information size [34, 35].This will result in delay avoidance in the process of information transfer, information compression and decompression in the format of codes as well as cost-effectiveness maintenance of implementing telemedicine technologies and reducing the payback period [하-38].

Thus the aims of this study is to select the type of Telecommunication services with regard to the desired interactive method in the infrastructure of telemedicine in such a way that the costeffectiveness is perfectly maintained by providing services through convergence of information size with the type of transferred information.

\section{MATERIAL AND METHODS}

As a quantitative type of research, this study was descriptive and cross-sectional, that was conducted in 2019.

The questionnaire used in this study consists of 45 questions in five classes. It examines the use of each of the nine tele-communication technology according to the interactive modes and communication services in the telemedicine platform.

The analysis was carried out thorough a questionnaire survey among 60 participants, including 30 information technology experts in educational hospitals affiliated to Tehran university of medical science, 15 faculty members holding Ph.D. degrees in the fields of Health Information Management and Medical Informatics and 15 Ph.D. students majoring in Medical Informatics from Tehran, Shahid Beheshti, as well as Iran University of Medical Sciences. In this study, the sampling methodology followed a simple non-random method.

Four questionnaires were designed to assess the amount of utility of each of the technologies for information transfer in the form of each of the four interactive methods. Likert rating scale (0-4) was employed to quantify each of the responses (very good, good, fair, poor, and very poor). Questionnaires' validity was confirmed, prior to distribution, through the recommendations of three experts in the field of Medical Informatics. In order to confirm the reliability of the questionnaires testretest was applied to four medical informatics experts, who were asked to repeat the questionnaire one week later. Finally, the validity of the test-retest was approved (0.85).

Followed by the distribution of the questionnaire among the population under study, data was collected and prepared for analysis. Likert rating scale was employed to quantify and subsequently analyze the information, using descriptive statistics principles with SPSS software. 


\section{RESULTS}

To determine the optimal telemedicine communication technologies with regards to network interactive modes, the study criteria were evaluated by 60 samples of attending experts through the decision Delphi technique. Table 1, shows the attending expert's socio- demographic characteristics.

Table 1: Socio-demographic characteristics of the samples

\begin{tabular}{|c|c|c|}
\hline Variable & Categories & Frequency \\
\hline \multirow{3}{*}{ Academic field } & $\begin{array}{l}\text { Information } \\
\text { technology }\end{array}$ & 14 \\
\hline & $\begin{array}{l}\text { Health } \\
\text { information } \\
\text { technology/ } \\
\text { management }\end{array}$ & 37 \\
\hline & $\begin{array}{l}\text { Medical } \\
\text { informatics }\end{array}$ & 9 \\
\hline \multirow{4}{*}{ Literacy rate } & $\mathrm{BS}$ & 24 \\
\hline & $\mathrm{MSc}$ & 6 \\
\hline & PhD candidate & 15 \\
\hline & $\mathrm{PhD}$ & 15 \\
\hline \multirow{2}{*}{ Gender } & Male & 38 \\
\hline & Female & 22 \\
\hline \multirow{4}{*}{$\begin{array}{l}\text { Age group } \\
\text { (years) }\end{array}$} & $20-30$ & 23 \\
\hline & $30-40$ & 19 \\
\hline & $40-50$ & 11 \\
\hline & $50<$ & 7 \\
\hline \multirow{4}{*}{$\begin{array}{l}\text { Work } \\
\text { experience } \\
\text { (years) }\end{array}$} & $<5$ & 16 \\
\hline & $5-10$ & 14 \\
\hline & $10-15$ & 21 \\
\hline & $15<$ & 9 \\
\hline
\end{tabular}

According to the results of Delphi surveys (Table 2), technologies with low bandwidth such as POTS and ISDN (56 Kbps and $128 \mathrm{Kbps}$, respectively; average of 4 and 4.10) are suitable technologies to send simple textual information in the format of Email and fax, i.e. Unicode and American Standard Code for Information Interchange (ASCII) formats. This textual information may include health status reports, clinical findings, physician's prescriptions, etc. These technologies, from the perspective of the participants were not mentioned among the ideal technologies for the transfer of larger information, such as multimedia, due to delay in sending information (averages of 0.96 and 2.60).

Regarding the transfer of textual-numerical laboratory finding and visual information such as radiological and pathological reports in the format of storage-and-forward technology, ISDN Basic Rate technology (128 Kbps, an average of 4), was known as the most ideal technology. This technology does not function efficiently in sending large-scale multimedia information, such as videos (average of 2).
Voice in telemedicine technology is exchanged as telephone conversations and as parts of the sounds of body organs, such as heart and lung (mp3 and WAV formats). From the perspective of the interviewees, the most ideal technology for sending voice in telemedicine is ISDN Primary Rate, ATM, ISDN / DSL, VDSL, HDSL and SDSL, with the averages of $4.06,4.16,4.05,4,4.45$ and 4.43 . It is worth pointing out that the average bandwidth required for the mentioned technologies is up to a few megabytes per second, except for ATM, which is a few gigabytes per second.

To send the information in video formats, ATM and VDSL technologies with ultra-high bandwidth (155 Mbps to $9 \mathrm{Mbps}$ ) were known to be the most ideal technologies (averages of 4.30 to 4.44). Other technologies, such as HDSL and SDSL and ADSL, with high bandwidth (1.5 to $8 \mathrm{Mbps}$ ) were identified as the ideal technology for sending video information (averages of 4, 4.21 and 4.59). All the above communication technologies, from the perspective of the respondents, were not mentioned as the costeffective technologies for sending textual information in email format (averages of 2.60, 2.93, $1.89,2.32$, and 2.22). Moreover, technologies of ATM, VDSL and HDSL technologies with the averages of 2.96, 2.30, and 2.78 were not identified as cost-effective technologies to send textual numerical information, such as laboratory findings and visual data, such as radiological and pathological reports in forward-storage format.

In the last section, for the exchange of information in hybrid modes :A combination of different media in the form of synchronized and asynchronous methods, DSL exchange technologies were considered by experts due to the ability to change their bandwidth according to the needs of the network traffic. Therefore, the sum of the means assigned to these technologies was greater than 4 (4.05 for VDSL, 4.69 for HDSL, 4.78 for SDSL and 4.96 for ADSL).

\section{DISCUSSION}

In a report entitled as "Return on Investment Analysis of the VISN1 Telehealth Program" which focuses on the duration of return on investment in telemedicine and the improvement of costeffectiveness of implementing this technology, an emphasis is placed on the importance of bandwidth management, to be carried out through the convergence of the type of information and the interactive methods using the desired bandwidth for information transfer. This convergence leads into the improvement of cost-effectiveness of using telemedicine. To this end, in order to maintain costeffectiveness of providing services, it is recommended to apply low-cost technologies with lower bandwidth to send small-scaled textual 
information, such as health status report, and smallscaled textual-numerical information, such laboratory findings. It may not be economical to implement expensive technologies to transfer smallscaled information, using ultra-high bandwidth [3941].

The aim of this study was the selection of telemedicine applications in virtual patients care to identify most common and effective interaction modes in terms of digital communication modalities (video calls, VOIP, Remote Monitoring of Patient (RMP) data such as images or physiologic parameters, social messenger and etc.), data templates (text, images, voice and video), and timing aspects (e.g., synchronous, asynchronous, and combined digital services).

In a report entitled as "Quality of Service (QoS) in High-Priority Applications", selection of suitable bandwidth is considered to be subject to the extent of tolerating communication technologies delay occurrence. For example, transactions related to storage-and-forward technology, such as email and fax, have high tolerance and transactions related to the technologies of transfer of voice over Internet protocol and transfer of video information in video teleconferencing format and remote surgery applications, and have lower tolerance in delay occurrence. In other words, this means that delay in sending information in transactions that have lower tolerance can be detrimental [는 $\underline{44}]$. As a result, the lower the degree of error tolerance, the higher the bandwidth should be for information transfer, while considering the issue's sensitivity. This highlights the need for the process of bandwidth management. Hence, it is suggested to allocate higher bandwidth technology for the simultaneous applications of telemedicine, such as teleconferencing in video or audio format, as well as more critical applications such as remote surgery in comparison to the asynchronous application of storage-and-forward technology. For example in this report, the required bandwidth for the fax and audio conversation transfer is $28 \mathrm{Kbps}$; for voice on the phone transfer is $64 \mathrm{Kbps}$; for text and text-image email transfer is $128 \mathrm{Kbps}$; for audio teleconferencing transfer is 1 Mbps; for video teleconferencing transfer is $5 \mathrm{Mbps}$; and finally it is $1 \mathrm{Gbps}$ for teleconferencing in critical applications, such as remote surgeries, which require high resolution display and virtual space creation [ $\underline{43}-\underline{45}]$.

The number of users is another issue, which affects the selection of bandwidth. If a system, as a backbone, provides a large number of users with remote consulting, it will be more preferable to employ high bandwidth communication technologies. Consequently, it is recommended to use low bandwidth communication technology for systems with a small number of users. In an article entitled as "Quality of Service in Telemedicine", ATM technology is introduced as the main backbone for those transactions in telemedicine, which provide services to a large number of users. This technology, as the main information thoroughfare for large companies, which provide telemedicine services, is capable of not only responding to a large number of users, but also they meet the quality criteria established in telemedicine $[\underline{44}, \underline{46}]$.

POTS technology, as a result of using ordinary telephone lines, is an affordable and exclusive technology with a low bandwidth (56 Kbps) which is not suitable for sending large-scaled information such as video and audio formats, since they will result in delay occurrence. This technology is suitable and cost-effective for sending small-scaled textual and numerical information $[\underline{47}, \underline{48}]$.

It is not necessary to have high-resolution quality to carry out face-to-face video conferencing for remote consultation, such as tele-psychology. To this effect, ISDN technology with a bandwidth of up to $2 \mathrm{Mbps}$, SDSL with a bandwidth of $2.3 \mathrm{Mbps}$ and HDSL with a bandwidth of $1.54 \mathrm{Mbps}$, are useful options for transporting multimedia information with medium resolution in storage-and-forward format. On the contrary, to perform remote surgeries, it is vital to have high-resolution display and the minimum delay occurrence in information transfer. Hence, expensive technologies that promise ultrahigh bandwidth should be employed. Thus, the most suitable technologies for information transfer are ATM with an ultrahigh bandwidth (155 Mbps to 9 Gbps) and VDSL technology with a maximum of 54 Mbps, which enable the transmission of multimedia information with maximum resolution and minimum delay. Accordingly, these technologies that promise ultrahigh bandwidth are suitable for remote surgery via video conferencing by virtual reality creation and robot control.

The results obtained from the application of ISDN to information transfer in telemedicine format, revealed that since in this communication technology the bandwidth variably ranges from 144 Kbps up to 52 Mbps in VDSL it may be inferred that VDSL is suitable for large-scaled multimedia data. However, HDSL, SDSL and ADSL with a medium bandwidth (2-8 Mbps) are more appropriate for those information transfer procedures that highresolution display is not an issue, such as smallscaled video and audio information. Finally, IDSL technologies (144 Kbps) are more suitable for transferring smaller-scaled information in the format of storage-and-forward and fax technologies, such as laboratory texts and data as well as health status reports.

Generally, it can be stated that the respondents in this study find the technologies with low bandwidth (a few tens of Kbps) more cost-effective to transfers 
mall-scaled information, such as texts and numerical data, through using storage-and-forward technologies, such as email and fax. This is the case while they do not find it suitable to use the same technologies for sending large-scaled information, i.e. multimedia, thus, they were rated low. Communication technologies, using an average bandwidth (a few Mbps) were considered to be functional for sending information with an average size, such as pathological images, radiological images, pieces of heartbeat sound and some video images in asynchronous mode. Finally, they found it appropriate to use the technologies that promise ultra-high bandwidth (several tens to hundreds of Mbps and even several Gbps) to send large-scaled information in multimedia format and with high quality with minimum delay and maximum synchronicity in sending information. It is worth pointing out that this technology is expensive; hence, careful attention needs to be paid to its purchase and the type of its application for the purpose of avoiding waste of money and maintaining costeffectiveness.

Table 2: Assessing the adoption of interactive modes and communication technologies for telemedicine services

\begin{tabular}{|l|c|c|c|c|c|}
\hline \multirow{2}{*}{$\begin{array}{l}\text { Technical } \\
\text { requirements }\end{array}$} & \multicolumn{5}{|c|}{ Interactive modes (Mean/Percentage) } \\
\cline { 2 - 6 } & $\begin{array}{l}\text { Text based store\& } \\
\text { forward technology }\end{array}$ & $\begin{array}{l}\text { Real-time } \\
\text { chatting }\end{array}$ & $\begin{array}{l}\text { Pre-recorded } \\
\text { audio \& video }\end{array}$ & $\begin{array}{l}\text { Real-time audio or } \\
\text { videoconference }\end{array}$ & Hybrid mode \\
\hline POTS & $4(80)$ & $3.30(66)$ & $1.82(36.4)$ & $0.96(19.2)$ & $1.06(21.2)$ \\
\hline ISDN Basic Rate & $4.10(82)$ & $4(80)$ & $3.10(62)$ & $2(40)$ & $1.88(37.6)$ \\
\hline ISDN Primary Rate & $3.60(72)$ & $3.34(66.8)$ & $4.06(81.2)$ & $3.96(79.2)$ & $2.66(53.2)$ \\
\hline ATM & $2.93(58.6)$ & $2.96(59.2)$ & $4.16(83.2)$ & $4.30(86)$ & $2.88(57.6)$ \\
\hline ISDN/DSL & $4(80)$ & $3.76(75.2)$ & $3.92(78.4)$ & $2.86(57.2)$ & $3.50(70)$ \\
\hline VDSL & $1.89(37.8)$ & $2.20(44)$ & $4(80)$ & $4.44(88.8)$ & $4.05(81)$ \\
\hline HDSL & $3(60)$ & $2.78(55.6)$ & $4.45(89)$ & $4(80)$ & $4.69(93.8)$ \\
\hline SDSL & $2.32(46.4)$ & $3.33(66)$ & $4.43(88.6)$ & $4.41(88.2)$ & $4.78(95.6)$ \\
\hline ADSL & $2.22(544.4)$ & $3.34(66.8)$ & $3.50(70)$ & $4.95(99)$ & $4.96(99.2)$ \\
\hline
\end{tabular}

\section{CONCLUSION}

Nowadays most remote care services especially in organizations providing telemedicine services encompasses s a range of services including noninteractive or asynchronous telemedicine counseling for non-critical situations to real-time or synchronous services to meet the emergency needs. In this situation selecting the communication technology that has variable bandwidth can meet the needs of different groups in different situations in according to their special interactive modes. Hence DSL technologies (e.g. ADSL, SDSL, and HDSL) have been proposed for hybrid platforms due to their wide bandwidth ranges or adoptive.Finally combining the functions of online conversation and real-time clinical data exchange technologies can provide technical support to the emerging need for workflow digitalization. Telemedicine provides a diversity of modalities affordable by patients and physicians including Telephone text messages (SMS), E-mail and web portals, secure Telephone calls or VOIP, video calls, interactive mobile health applications (m-Health), RMP, and video-conference.

\section{ACKNOWLEDGMENTS}

We thank the Research Deputy of Abadan Faculty of Medical Sciences for financially supporting this project. We also would like to thank all experts who participated in this study and played a role in the validation of the data elements.

\section{AUTHOR'S CONTRIBUTION}

The authors agree on this final form of the manuscript, and attested that all authors contributed in the final draft of the manuscript.

\section{CONFLICTS OF INTEREST}

The authors declare no conflicts of interest regarding the publication of this study.

\section{FINANCIAL DISCLOSURE}

This article is extracted from a research project supported by Abadan Faculty of Medical Sciences (IR.ABADANUMS.REC.1398.099).

\section{REFERENCES}

1. Ho HJ, Lim WY, Ang B, Chow A. Use of surveillance technology to enhance exposure management for healthcare workers during the COVID-19 pandemic. J Hosp Infect. 2021; 107: 101-2. PMID: 32980491 DOI:

\subsection{6/j.jhin.2020.09.024 [PubMed]}

2. Liu WI. New opportunities for healthcare driven by smart technology. Hu Li Za Zhi. 2020; 67(5): 4-5. PMID: 32978759 DOI: 10.6224/JN.202010_67(5).01 [PubMed] 
3. Baillieu R, Hoang H, Sripipatana A, Nair S, Lin SC. Impact of health information technology optimization on clinical quality performance in health centers: A national cross-sectional study. PLoS One. 2020; 15(7): e0236019. PMID: 32667953 DOI: 10.1371/journal.pone.0236019 [PubMed]

4. Giess CS, Wang A, Frost EP, Chikarmane SA, Boland GW, Khorasani R. Impact of an information technology- Enabled quality improvement initiative on timeliness of patient contact and scheduling of screening mammography recall. AJR Am J Roentgenol. 2019; 213(4): 880-5. PMID: 31268733 DOI: 10.2214/AJR.19.21397 [PubMed]

5. Edge C, George J, Black G, Gallagher M, Ala A, Patel S, et al. Using telemedicine to improve access, cost and quality of secondary care for people in prison in England: A hybrid type 2 implementation effectiveness study. BMJ Open. 2020; 10(2): e035837. PMID: 32075846 DOI: 10.1136/bmjopen2019-035837 [PubMed]

6. Romanick-Schmiedl S, Raghu G. Telemedicine maintaining quality during times of transition. Nat Rev Dis Primers. 2020; 6(1): 45. PMID: 32483168 DOI: $10.1038 / \mathrm{s} 41572-020-0185-x$ [PubMed]

7. Zhang W, Cheng B, Zhu W, Huang X, Shen C. Effect of telemedicine on quality of care in patients with coexisting hypertension and diabetes: A systematic review and meta-analysis. Telemed J E Health. 2020. PMID: 32976084 DOI: $10.1089 /$ tmj.2020.0122 [PubMed]

8. Evans L, Mohamed B, Thomas EC. Using telemedicine and wearable technology to establish a virtual clinic for people with Parkinson's disease. BMJ Open Qual. 2020; 9(3): e001000. PMID: 32958473 DOI: 10.1136/bmjoq-2020-001000 [PubMed]

9. Jenkins J, Oyama 0 . Telemedicine: The art of innovative technology in family medicine. Int $\mathrm{J}$ Psychiatry Med. 2020; 55(5): 341-8. PMID: $32883143 \quad$ DOI: $10.1177 / 0091217420951038$ [PubMed]

10. Pellegrini D, Torlasco C, Ochoa JE, Parati G. Contribution of telemedicine and information technology to hypertension control. Hypertens Res. 2020; 43(7): 621-8. PMID: 32203451 DOI: 10.1038/s41440-020-0422-4 [PubMed]

11. Law T, Cronin C, Schuller K, Jing X, Bolon D, Phillips B. Conceptual framework to evaluate health care professionals' satisfaction in utilizing telemedicine. J Am Osteopath Assoc. 2019; 119(7): 435-45. PMID: 31233109 DOI: $10.7556 /$ jaoa.2019.080 [PubMed]

12. Tsioumanis V, Mangita A, Diomidous M. Applications and developments of telemedicine in Greece. Stud Health Technol Inform. 2016; 226: 253-5. PMID: 27350518 [PubMed]

13. Nalugo M, Craner DR, Schwachter M, Ponsky TA. What is "telemedicine" and what does it mean for a pediatric surgeon? Eur J Pediatr Surg. 2014; 24(4): 295-302. PMID: 25111277 DOI: 10.1055/s-00341386647 [PubMed]

14. Rao SS, Loeb AE, Amin RM, Golladay GJ, Levin AS,
Thakkar SC. Establishing telemedicine in an academic total joint arthroplasty practice: Needs and opportunities highlighted by the COVID-19 pandemic. Arthroplast Today. 2020; 6(3): 617-22. PMID: 32328510 DOI: 10.1016/j.artd.2020.04.014 [PubMed]

15. Hakim AA, Kellish AS, Atabek U, Spitz FR, Hong YK. Implications for the use of telehealth in surgical patients during the COVID-19 pandemic. Am J Surg. 2020; 220(1): 48-49. PMID: 32336519 DOI: 10.1016/j.amjsurg.2020.04.026 [PubMed]

16. Smith WR, Atala AJ, Terlecki RP, Kelly EE, Matthews CA. Implementation guide for rapid integration of an outpatient telemedicine program during the COVID19 pandemic. J Am Coll Surg. 2020; 231(2): 216-22. PMID: $32360960 \quad$ DOI: 10.1016/j.jamcollsurg.2020.04.030 [PubMed]

17. Li W, Che ZJ, Li YW. Research on the congestion control of broadband integrated service digital network based on ATM. International Conference on Machine Learning and Cybernetics. IEEE; 2006.

18. Mupparapu M. Voice over Internet protocol for the orthodontic practice: A sensible switch from plain old telephone service. Am J Orthod Dentofacial Orthop. 2008; 133(3): 470-5. PMID: 18331949 DOI: 10.1016/j.ajodo.2006.09.048 [PubMed]

19. Vodrazka J, Jares P. Selection of digital subscriber lines ready for next generation access. Advances in Electrical and Electronic Engineering. 2015; 13(4): 327-31.

20. Prahmkaew S, Pongpadpinit S, Viriyaphol P, Nukoon CJ. Performance evaluation of adaptive rate control over uncompressed high-definition content transmission with paralleled digital subscriber lines. International Conference on Hybrid Information Technology. Springer; 2015.

21. Altinkemer K, Bose I. Asynchronous transfer mode networks with parallel links and multiple service classes. European Journal of Operational Research. 2003; 146(1): 181-98.

22. Robertazzi T, Robertazzi T. Basics of computer Networking. Springer; 2012.

23. Andrikopoulos I, Chuberre N, Cohen M, Courseille O, Duval R, Farineau J, et al. An overview of digital video broadcasting via satellite services to handhelds (DVB-SH) tnchnology. IEEE; 2008.

24. Courseille O, Poire P, Durand MC, Mazzella M. Integrating satellite digital radio broadcasting (SDB), terrestrial cellular technology and EGNOS satellite navigation: The RELY project. In: Gayraud T, Mazzella M, Boavida F, Monteiro E, Orvalho J [eds]. Broadband satellite communication systems and the challenges of mobility. Springer; 2004.

25. Yu QT, Peng HF. Target detection technology in passive radar based on broadcasting satellite signals. International Conference on Computer Science and Mechanical Automation. IEEE; 2015.

26. Dafonte C, Gomez A, Arcay B, Taboada JA. Intelligent management of processes in a ICU telemedicine system. International Conference of the IEEE 
Engineering in Medicine and Biology Society. IEEE; 2000.

27. Mink BF, Mink KM. Real time multispecialty telehealth interactive patient wellness portal (IPWP). Google Patents; 2017.

28. McLendon SF. Interactive video telehealth models to improve access to diabetes specialty care and education in the rural setting: A systematic review. Diabetes Spectr. 2017; 30(2): 124-36. PMID: 28588379 DOI: $10.2337 /$ ds16-0004 [PubMed]

29. Hickey S, Gomez J, Meller B, Schneider JC, Cheney M, Nejad S, et al. Interactive home telehealth and burns: A pilot study. Burns. 2017; 43(6): 1318-21. PMID: $28641914 \quad$ DOI: $\quad 10.1016 /$ j.burns.2016.11.013 [PubMed]

30. Gur M, Nir V, Teleshov A, Bar-Yoseph R, Manor E, Diab G, et al. The use of telehealth (text messaging and video communications) in patients with cystic fibrosis: A pilot study. J Telemed Telecare. 2017; 23(4): 489-93. PMID: 27177869 DOI: 10.1177/1357633X16649532 [PubMed]

31. Nord G, Rising KL, Band RA, Carr BG, Hollander JE. On-demand synchronous audio video telemedicine visits are cost effective. Am J Emerg Med. 2019; 37(5): 890-4. PMID: 30100333 DOI: 10.1016/j.ajem.2018.08.017 [PubMed]

32. Wade VA, Karnon J, Elshaug AG, Hiller JE. A systematic review of economic analyses of telehealth services using real time video communication. BMC Health Serv Res. 2010; 10: 233. PMID: 20696073 DOI: $10.1186 / 1472-6963-10-233$ [PubMed]

33. Reed ME, Parikh R, Huang J, Ballard DW, Barr I, Wargon C. Real-time patient-provider video telemedicine integrated with clinical care. $\mathrm{N}$ Engl J Med. 2018; 379(15): 1478-9. PMID: 30304654 DOI: 10.1056/NEJMc1805746 [PubMed]

34. Sarmah S, Sarma SK. A novel approach to prioritized bandwidth management in 802.11e WLAN. International Conference for Convergence in Technology. IEEE; 2019.

35. Rafiq A, Hayat MF. Bandwidth utilization and management algorithms (BUMAs) for NG-EPON. Journal of Network and Systems Management. 2020; 28(4): 1522-46.

36. Harnett B. Telemedicine systems and telecommunications. J Telemed Telecare. 2006; 12(1): 4-15. PMID: 16438772 DOI: 10.1258/135763306775321416 [ PubMed]

37. Rodas E, Mora F, Tamariz F, Cone SW, Merrell RC. Low-bandwidth telemedicine for pre- and postoperative evaluation in mobile surgical services.
J Telemed Telecare. 2005; 11(4): 191-3. PMID: $15969794 \quad$ DOI: $\quad 10.1258 / 1357633054068919$ [PubMed]

38. Aoki N, Dunn K, Johnson-Throop KA, Turley JP. Outcomes and methods in telemedicine evaluation. Telemed J E Health. 2003; 9(4): 393-401. PMID: 14980098 DOI: $10.1089 / 153056203772744734$ [PubMed]

39. Moore M, Moreschi A, Rieger K, Vardaro M. Return on investment analysis of the VISN1 telehealth program [BS Project]. Worcester Polytechnic Institute. Massachusetts; 2013.

40. Crowley ST, Belcher J, Choudhury D, Griffin C, Pichler $\mathrm{R}$, Robey B, et al. Targeting access to kidney care via telehealth: The VA experience. Adv Chronic Kidney Dis. 2017; 24(1): 22-30. PMID: 28224939 DOI: 10.1053/j.ackd.2016.11.005 [PubMed]

41. Andren JC. A framework to improve enterprise-wide implementations: The case of the veterans health administration Telehealth expansion [MSc Thesis]. Massachusetts Institute of Technology; 2013.

42. Nageba E, Fayn J, Rubel P. A model driven ontologybased architecture for supporting the quality of services in pervasive telemedicine applications. International Conference on Pervasive Computing Technologies for Healthcare. IEEE; 2009.

43. Algaet MA, Noh ZAB, Shibghatullah AS, Milad AA, MustaphaA. Provisioning quality of service of wireless telemedicine for e-health services: A review. Wireless Personal Communications. 2014; 78: 375406.

44. Nikolaidis Y, Efthymiadis G, Angelidis P. Quality assessment of a second opinion telemedicine service. Health and Technology. 2019; 9(5): 659-78.

45. Cason J, Behl D, Ringwalt S. Overview of states' use of telehealth for the delivery of early intervention (IDEA Part C) services. Int J Telerehabil. 2012; 4(2): 39-46. PMID: 25945202 DOI: 10.5195/IJT.2012.6105 [PubMed]

46. Nanda P, Fernandes RC, editors. Quality of service in telemedicine. International Conference on the Digital Society. IEEE; 2007.

47. Tischer S, Kleinfelter K. Systems and methods for passing through alternative network device features to plain old telephone system (POTS) devices. Google Patents; 2009.

48. Tyroler D, Orlando RJ, Rothman RK, Jordan R, Lizza AM. Wireless interface device allowing a reliable digital and audio communication transfer between a security system, POTS and/or IP network modem device. Google 2015. 